\title{
EXPERIMENTAL STRESS ANALYSIS AND FEA OF DENTAL IMPLANTS
}

\author{
S. P. Gosavi ${ }^{1}$, P. N. Dhatrak ${ }^{2}$, K. M. Narkar ${ }^{3}$ \\ ${ }^{1}$ Student, Department of Mechanical Engineering, D. Y. Patil College of Engineering, Maharashtra, India \\ ${ }^{2}$ Assistant Professor, Department of Mechanical Engineering, MIT College of Engineering, Maharashtra, India \\ ${ }^{3}$ Assistant Professor, Department of Mechanical Engineering, D. Y. Patil College of Engineering, Maharashtra, India
}

\begin{abstract}
Dental implants are used as prosthetic treatment alternatives made of Titanium for treating partial edentulism in patients. The oessointegration of bone and implant at the interface is of utmost importance as the success or failure of a dental implant depends on the manner in which stresses are transferred to the surrounding bone. The osseointegrated dental implant plays a role similar to that of natural teeth as it is exposed to static and dynamic loadings continuously. However, the functional forces in Osseointegrated dental implant are transmitted directly to the jaw bone as compared to the natural teeth where there is a healthy periodontium. This could cause micro-fracture at the bone-implant interface, fracture of implant, loosening of components of implant system and unwanted bone resorption. Therefore, it is essential to understand stress concentration on implants at the bone implant interface. This study aims in investigating and monitoring the stresses along the bone implant interface for different types of dental implant.Photo elastic stress analysis was carried on four commercial implants with varying diameter and same length, and the verification of the experimental results was done using finite element analysis.
\end{abstract}

Keywords: Dental Implant, Photoelasticity, Stress Analysis, Implant Bone Interface, FEA.

\section{INTRODUCTION}

Dentistry plays a important role in replacing missing teeth in form of dental implants. Dental implants are insertions into a patient's mouth bone to which an artificial tooth crown is attached. The bonding between the implant and bone must be rigid enough to bear the stress due to different loading conditions. Design and Insertion technique are the two important factors which determine success of implant. Implant according to invention seeks mechanical properties and stress distribution in bone that are similar to those of natural tooth.

Excessive stress transferred from implant to bone may prone to damage .Hence for a better bone implant interfacing threaded part of implant must be self-tapping for better integrity and increase in contact surface area. Many factors have been found to influence this interfacial bonding between the implant and bone and thus the success of implants.

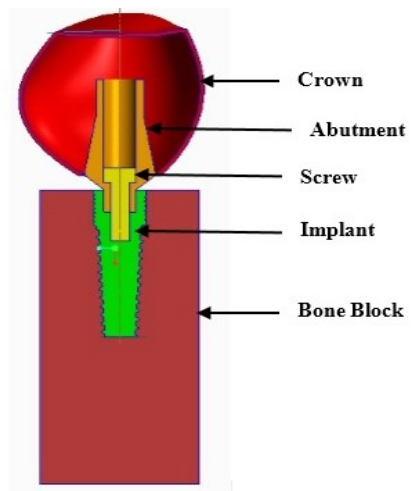

Fig.1 - Components of Implant

\section{LITERATURE REVIEW}

Saluja et al studied the effect of length and diameter on stress distribution by creating a $3 \mathrm{D}$ solid model of Indigeneous titanium dental implant and mandible and subjecting to FEA using Ansys. Implant length does not affect Stress concentration and distribution. Increase in diameter increases surface contact area thus making implant stable and reducing stress pattern [1]. Arsalanlo et al investigated the effect of different thread types on loading in implant abutment interface. Models with varying diameter and length were analysed using FEA to understand stress distribution They found that increase in thread depth enhances stress distribution on the other hand decrease in thread pitch reduces implant stability.[2]

Zhihong Mao evaluated the effect of abutment \& fixed screw on dental implant system by modeling of three screwtype dental implant systems with three fixed screw diameter sizes and subjected to static occlusal force of $100 \mathrm{~N}$ with $15^{\circ}$ for FEA. Stress increases with improper size of abutment \& fixed screw [3]. Giuseppe et al evaluated the influence of implant design parameters such as diameter, thread type, length on load transfer mechanism of oessointegration in dental implant by analyzing and comparing ten different types of implants (varying length, diameter, thread shape and geometry) .He concluded that such as implant diameter, length, thread shape highly influence design parameters in load transfer on Implant [4].

Ghorpade et al carried a literature survey to analyse role of design parameters related to bone -implant interface on stress distribution in FEA and experimental studies. 
Modeling approach and implant geometry are influential factors for the accuracy of analysis [5]. Ausiello et al investigated influence of implant design factors for implant longevity.DOE approach was adopted for automatic generation of different implant designs and analysis using FEA. Implant stability is dependent on thread-width and thickness by correlating design parameters with each other [6]. Desai et al determined stresses in bone implant interface using various dental implant designs. Modeling and analysis of eight types of implants with different thread designs to evaluate stresses and strains around the implants [7]. Bahrami et al evaluated stress distribution in bone implants interface and dental prosthesis. More thread depth to increase contact surface area can reduce stresses in surrounding bone [8]. Szajek et al Presentation of an optimization procedure for minimizing diameter of twocomponent implantology system. Two component implant model based on FE analysis was optimized and incorporated into the hybrid optimization procedure (genetic and HookeJevees algorithm). The presented hybrid technique optimized diameter without disturbing the defined boundaries [9]. Mohammed et al studied stress distribution in dental implants by modeling and analysis of different implant designs by varying length and diameter subjecting to $50 \mathrm{~N}$ tensile, $100 \mathrm{~N}$ compressive, $20 \mathrm{~N}$ bending. Implant diameter and length, crestal bone geometry and placement site affect the load transfer mechanism [10].

Kong et al evaluated the cylinder implant thread height and width using 3D finite element analysis. He concluded that in design of a screw type implant optimum thread height is a more important factor than thread width for reduction of stress in bone [11]. Mansour et al in his work to studied stress distribution around tapered and cylindrical threaded implant geometries using three-dimensional finite element stress analysis [12]. Baggi et al studied to analyze the influence of implant diameter and length on stress distribution. Implant shape, geometry and bone resorption possibly affect the load transfer mechanism leading to failure [13].

Implant geometry is an important objective in biomechanical optimization of dental implants. It is necessary to evaluate thread design of dental implant. From the above literature review study the following conclusions can be drawn for the stability and longevity of Dental Implant:

- The durability as well as the stability of Dental implant depends on oessointegration which is solely dependent on the bone implant interface. Hence bone implant interface is one of the regions to be considered.

- Secondly the use of the threaded implant may enlarge the contact area between the implant and bone and help dissipating interfacial stresses

- There has been insufficient research focusing on the pattern of load transfer and the failures with the effect of implant length correlated with the force transmission area of implant bone interface.

\section{MATERIALS AND METHODOLOGY}

This study was performed on four different implants of diameter $3.3,3.5,3.75$ and 4.2 with $10 \mathrm{~mm}$ length each in following steps which included photoelastic stress analysis, creating solid models of implants, creating finite element model and analyzing the process of load transfer and stress distribution.

\subsection{Photo elastic Model Creation And Photo}

\section{elasticity}

Epoxy resins are most commonly used model material for photoelasticity experiment. Photoelastic Model blocks of dimensions $10 \times 20 \times 30 \mathrm{~mm}$ are prepared. The crown used for our study is Porcelain Fused to Metal type (PFM). The shape of the crown is same for all the system.

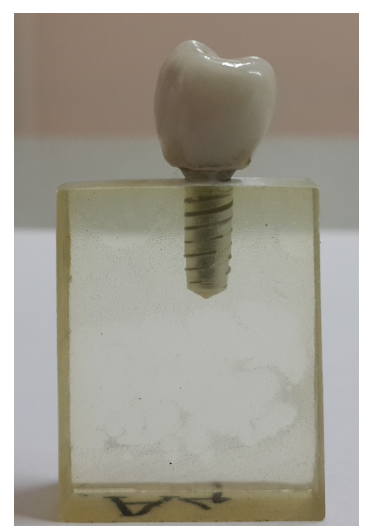

Fig.2 - Photoelastic Model

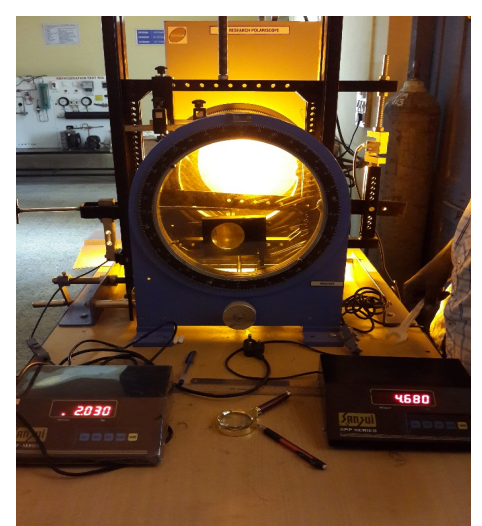

Fig.3 - Photoelasticity Set-up
Photoelastic model differs in mechanical properties from actual prototype. Hence the actual service load cannot be directly applied on photoelastic model for testing. Determination of loads to be applied on model can be done simply with comparison of Young's modulus of the two materials. In case of photoelastic model being Araldite $(\mathrm{E}=3300 \mathrm{Mpa})$ and actual prototype being composed of cancellous and cortical bone $(\mathrm{E}=1370 \mathrm{Mpa}$ and $E=13400 \mathrm{Mpa}$ ) load applied on model need to be magnified were calculated.

The experimental setup consists of a loading frame, light source i.e. monochromatic as well as white light, Polarizer and Analyzers Plates, Quarter wave Plates and a load cell. Loading indicator can be adjusted in finite number of step in both horizontal and vertical planes using hole arrangement provided, depending upon size of model.

The analysis was made using a circular polariscope, an analyzer, and a source of Yellow light to produce internal pattern stresses in the photoelastic material. The abutment dental implants are screwed to acrylic blocks by drilling and screwing method of the implantation.

\subsection{Modeling The Implant And Surrounding Bone}

3D CAD models of four dental implants were modelled. This was done to study the stress distribution in implant 
screw for varying values of diameter. A bone block analogous to tooth bone comprising of cancellous region surrounded by $2 \mathrm{~mm}$ thick cortical region was created.

The material of the implant was assumed to be isotropic and linearly elastic. The implant, abutment, and abutment screw were all designed to be titanium alloy.

Two types of bone density were modeled by varying the elastic modulus of cortical bone and cancellous bone (with high and low densities). The following material properties were considered [13].

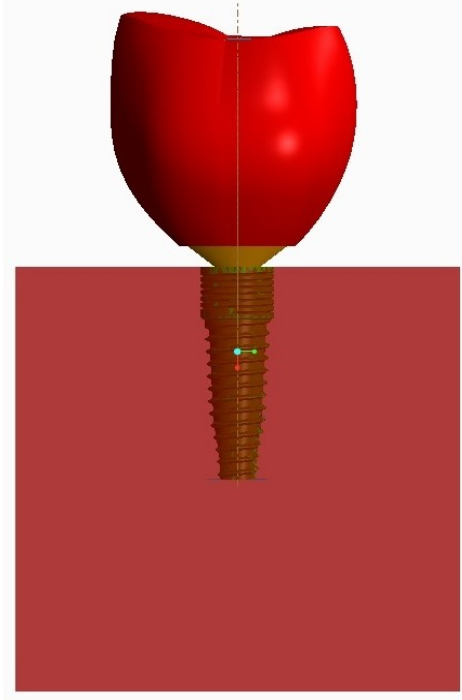

Fig. 4 - Assembled Model I

\subsection{MATERIAL PROPERTIES}

Table. 1 - Material Properties

\begin{tabular}{|l|l|l|l|}
\hline Material & Component & $\begin{array}{l}\text { Modulus of } \\
\text { Elasticity } \\
(\mathrm{MPa})\end{array}$ & $\begin{array}{l}\text { Poisson's } \\
\text { Ratio }(\mu)\end{array}$ \\
\hline Titanium & Implant & \multirow{2}{*}{113000} & 0.33 \\
\cline { 2 - 2 } & Abutment & & \\
\cline { 2 - 4 } & Screw & 13400 & 0.26 \\
\hline Cortical & Cortical & 1370 & 0.31 \\
\hline Cancellous & Cancellous & 70000 & 0.3 \\
\hline Porcelain & Crown & \multicolumn{2}{|l}{} \\
\hline
\end{tabular}

\subsection{Loading And Boundary Condition}

The dental implant system is treated as isotropic homogeneous elastic material. A combination of static vertical load of $116 \mathrm{~N}$ and a lateral load of $40 \mathrm{~N}$ was applied on the implant system [13] [14]. The material of the implant was assumed to be isotropic and linearly elastic.

As the implant system and the cancellous bone embedded inside the cortical bone are supported at the bottom by the mandibular bone, hence the bottom of the prototype was fixed with all degrees of freedom.

\subsection{MESHING OF MODELS}

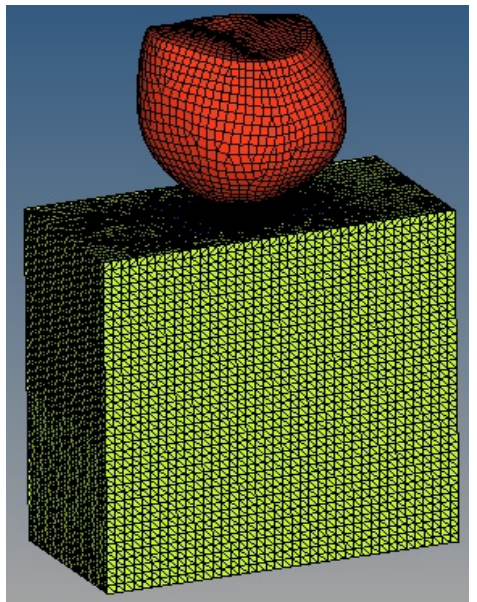

Fig.5 - Meshed Model I

The elements used in meshing of all three-dimensional models are Tetrahedral element. The implant, cancellous and cortical bone were meshed with mesh convergence criteria.

Meshing was done for all models as shown in fig.5

\subsection{FEA OF THE MODELS}

The Finite Element Linear static analysis was performed using ANSYS software to analyze the stress distribution in the cancellous region along the bone implant interface. The stress intensity results are shown for model I in fig.

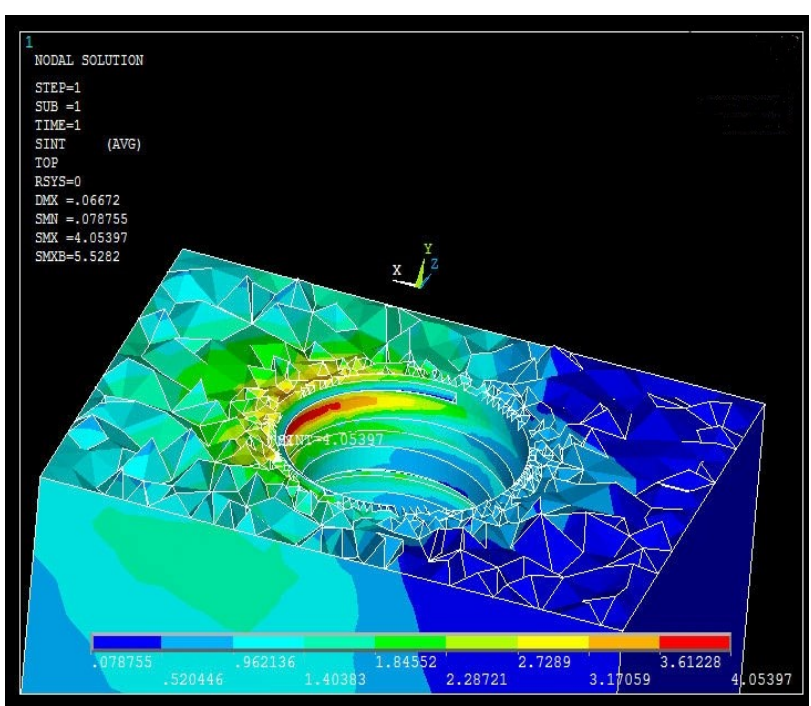

Fig.5 -Stress distribution in cancellous bone in Model I

Fig. 6 shows the stress intensity value of $4.05 \mathrm{MPa}$ in Model I along the bone implant interface. The stress values were measured in neck region in cancellous bone. Stress contours for cancellous bone elements nearest the cortical boneimplant interface can be displayed for further verification that increasing implant diameter within morphological limits, decreases stress magnitudes at the cancellous boneimplant interface. The FEA results for other models are given below. 
Table 2 - Stress Intensity in Models Using FEA

\begin{tabular}{|l|l|}
\hline Model & Stress Intensity $(\mathrm{MPa})$ \\
\hline Model I & 4.05 \\
\hline Model II & 3.24 \\
\hline Model III & 3.47 \\
\hline Model IV & 4.13 \\
\hline
\end{tabular}

\section{EXPERIMENTAL RESULTS}

The Experimental photoelasticity results obtained are shown in table 2 .The material fringe constant for the acrylic material was obtained using disc calibration. Fringe pattern in each model on application of axial and compressive load are as shown in fig below.

Table 3 - Stress Intensity in Models

\begin{tabular}{|l|l|l|}
\hline Model & No. of fringes & Stress Intensity (MPa) \\
\hline Model I & 10 & 4.81 \\
\hline Model II & 5 & 3.93 \\
\hline Model III & 6 & 3.42 \\
\hline Model IV & 6 & 4.00 \\
\hline
\end{tabular}

\section{RESULT COMPARISON}

Maximum stress intensity was observed in the neck region along the cancellous bone implant interface. The FEA and the experimental results are shown in Table 4.

Table 4 - Stress Intensity in Models using FEA

\begin{tabular}{|l|l|l|}
\hline \multirow{2}{*}{ Models } & \multicolumn{2}{|l|}{ Stress Intensity (MPa) } \\
\cline { 2 - 3 } & Experimental & FEA \\
\hline Model I & 4.81 & 4.05 \\
\hline Model II & 3.93 & 3.24 \\
\hline Model III & 3.42 & 3.47 \\
\hline Model IV & 4.00 & 4.13 \\
\hline
\end{tabular}

\section{CONCLUSION}

After the completion of experimental and finite element analysis it was found that

1. For all models maximum stress was concentrated around neck region in the cancellous bone.

2. The results of FEA and experimental photoelasticity were equivalent.

3. With increase in the diameter of the implant the stress can be minimized.

\section{REFERENCES}

[1]. Bobin Saluja, MasoodAlam, T Ravindranath, A Mubeen et.al, Effect of length and diameter on stress distribution pattern of INDIDENT dental implants by finite element analysis , Journal of Dental Implants, Vol 2 , Issue 1, PP 19-25, 2012.

[2]. Zeinab Arsalanloo, Reza Telchi, and Kambiz Ghaemi Osgouie, Selection of Optimum Thread Type in Implants to Achieve Optimal Biomechanical Properties by Using 3D Finite Element Method, International Journal of Bioscience, Biochemistry and Bioinformatics, Vol. 4, Issue 3, PP 185 - 190,May
2014

[3]. Zhihong Mao, Nanning, Design of dental implant system by FEA, 2014 IEEE Workshop on Electronics, Computer and Applications, 978-1-4799-4565-8, PP 675-677, 2014.

[4]. Giuseppe Vairo and Gianpaolo Sannino, Comparative Evaluation of Osseointegrated Dental Implants Based on Platform - Switching Concept : Influence of Diameter, Length, Thread Shape, and In-Bone Positioning Depth on Stress-Based Performance, Computational and Mathematical Methods in Medicine ,Volume 2013, Article ID 250929,PP 1-15,2013.

[5]. Ratnakar R. Ghorpade , Shailesh S. Yelekar, Computational And Experimental Studies In Threaded Dental Implant Research, e-Journal of Dentistry, Vol 3, Issue 4, PP 457 -465, Oct - Dec 2013

[6]. Pietro Ausiello, Pasquale Franciosa , Massimo Martorelli , David C. Watts, Effects of thread features in osseo-integrated titanium implants using a statisticsbased finite element method ,ELSEVIER, Dental materials , Issue 28,PP 919-927(2012).

[7]. S.R Desai, MS Desai, G Katti, I Karthikeyan , Evaluation Of Design Parameters Of Eight Dental Implant Designs: A Two-Dimensional Finite Element Analysis, NJPC Vol. 15,Issue 2,PP 176-181, 2012

[8]. Babak Bahrami, Farzan Ghalichi, Finite Element Analysis of Stress Distribution in Immediately Loaded Dental Implant ,International conference of Biomedical Engineering,Tehran,Iran,21-22 Dec, 2012.

[9]. Krzysztof Szajek, Marcin Wierszycki and Tomasz Łodygowski, Reduction of the tooth-implant components dimensions by optimization procedure, CMM-2011 - Computer Methods in Mechanics, Vol.4, 9-12 May 2011.

[10]. Mohamed I. El-Anwar, Mohamed M. El-Zawahry, A Three Dimensional Finite Element Study On Dental Implant Design,Journal of Genetic Engineering and Biotechnology, Issue 9, PP 77-82, 2011.

[11]. Liang Kong, Kaijin Hu, Dehua Li, Yingliang Song, Evaluation of Cylinder Implant Thread Height and Width - 3D Finite Element Analysis, The International Journal of Oral and Maxillofollical Implants, Volume 23,Number 1,PP 65-74,2008.

[12]. Luigi Baggi, Ilaria Cappelloni, Michele Di Girolamo, Franco Maceri, and Giuseppe Vairo, The influence of implant diameter and length on stress distribution of osseointegrated implants related to crestal bone geometry: A three dimensional finite element analysis, The Journal of Prosthetic Dentistry, Volume 10, Issue 6, pp 423-431, Dec 2008.

[13]. N. Djebbar, B. Serier, B. Bachir Bouiadjra, S. Benbarek, A. Drai, Analysis of the effect of load direction on the stress distribution in dental implant, Materials and Design 31 Elsevier, pp 2097-2101, 2010.

[14]. Iniyan Thiruselvam N, Dhananjay M. Kulkarni and Savio D.S.A Lourenco, Preoperative simulation of implant angulation effect on mandibular masticatory stresses- a finite element study, Procedia Engineering, Vol.64, pp. 8145-824, 2013. 